\title{
Impact of Growing Area and Technological Aspects on Lebanese Olive Oil: Characterization by Unsupervised Methods
}

\author{
Omar H. Dib ${ }^{1,2}$, Ali Bassal ${ }^{2}$, Hussein Dib ${ }^{2}$, Rita Yaacoub ${ }^{2}$, Nathalie Locquet ${ }^{1}$, Luc Eveleigh $^{3}$ \& Christophe B. Y. \\ Cordella ${ }^{1}$ \\ ${ }^{1}$ UMR PNCA, AgroParisTech, INRA, Université Paris-Saclay, Paris, France \\ ${ }^{2}$ Department of Food Science and Technology, Lebanese University, Beirut, Dekweneh, Lebanon \\ ${ }^{3}$ Ingénierie Procédés Aliments, INRA, AgroParisTech, Université Paris-Saclay, Massy, France \\ Correspondence: Omar H. Dib, UMR PNCA, AgroParisTech, INRA, Université Paris-Saclay, Paris, France. Tel: \\ 961-382-1834. E-mail: dibhomar@gmail.com
}

Received: January 28, 2020

Accepted: March 2, 2020 Online Published: March 26, 2020

doi:10.5539/jfr.v9n2p48

URL: https://doi.org/10.5539/jfr.v9n2p48

\begin{abstract}
The influence of growing-area and technological aspects on the Lebanese Soury variety is presented. The pedoclimatic conditions, quality parameters, and fatty acids of oil samples were examined using chemometric tools to demonstrate the typicality of Lebanese olive oil. Furthermore, the effect of several technological factors on olive oil quality was studied. Accordingly, olive oil samples were collected across Lebanon corresponding to two climatic zones (Zone A: Low-altitude regions; Zone B: High-altitude regions). Principal component analysis was capable of discriminating the two zones with distinct fatty acid profiles. Zone A exhibited a fatty acid profile mostly dominated by linoleic, linolenic, palmitic, and palmitoleic acid content. As for zone B, it showed a unique fatty acid profile distinguished by oleic acid. These results are mostly attributed to the climatic effect and the altitude of the growing area. Moreover, independent component analysis, coupled to one-way ANOVA, demonstrated that significant differences $(\mathrm{p}<0.05)$ were found in quality indices (acidity and peroxide value) as well as fatty acid contents (oleic and linoleic acid) when comparing uncontrolled to controlled extraction methods. This study provides a baseline for future intensive characterization of Lebanese olive oil and detects the non-compliances attributed to the disqualification of olive oil virginity.
\end{abstract}

Keywords: chemometric tools, fatty acid, growing area, olive oil, quality assessment

\section{Introduction}

Olive trees cover $5.4 \%$ of the Lebanese territory or $8 \%$ of total agricultural lands in Lebanon (Investment Development Authority in Lebanon [IDAL], 2017). Olive groves, almost solely rain-fed, are dispersed over the provinces of Lebanon, producing 24,000 tons of olive oil per year ( International Olive Council [IOC], 2018). This production is being conducted by 485 registered mills scattered throughout Lebanon. About $85 \%$ of the mills still rely on traditional-extraction systems, particularly pressing method (Lebanese Ministry of Agriculture [MOA], 2012).

Granite millstones and nylon fiber mats, the basic components of the pressing mill, are still being used despite their high susceptibility to contamination, and most prominently, their effect on olive oil components (Giovacchino, 2000). Olive paste remnants on mats can initiate several biological and chemical processes affecting the virginity of olive oil. Olive quality may deteriorate by other technological variables occurring before and after processing. Irrational practices during fruit transport and olive and olive oil storage may lead to the lipolysis of triglycerides and to the chemical oxidation of fatty acids affecting the major constituents of olive oil (Gharbi et al., 2015).

Besides the technological aspect, a wide variety of factors such as olive cultivar (Aguilera et al., 2005), fruit maturity (Amanpour et al., 2019), and growing area (Arslan et al., 2013) have a significant influence on olive oil chemical composition. Among these factors, the effect of the growing area is undeniably of primary importance, especially on the fatty acid profile (Aguilera et al., 2005). In most studies, altitude and climate have a vast impact on the profile of fatty acids. For example, countries close to the equator tend to have a higher palmitic, palmitoleic, linoleic and alpha-linolenic concentrations in contrary to those with a very cool climate where a 
higher oleic level is observed (Aparicio et al., 1994). Furthermore, differences between regions with distinct altitudes were noticed, as higher altitudes increase monounsaturated fatty acids, and lower altitudes increase polyunsaturated and saturated fatty acids (Di Bella et al., 2007).

Lebanon's diverse topographies, stretching mainly in a north-south direction, and Mediterranean climate establishes the potential for fatty acid profiles discrimination. Studies targeting the fatty acid composition of Lebanese olive oil originating from different growing areas are scarce. Still, some authors have indicated the possibility that the pedoclimatic conditions of some Lebanese olive growing areas can influence both saturated and monounsaturated fatty acids (Merchak et al., 2017; Riachy et al., 2018).

This study investigates the use of chemometrics to highlight the effect of growing area on the Lebanese olive oil based on the resulting physicochemical parameters (mainly fatty acids) and to detect the technological variables leading to disqualification of Lebanese olive oil virginity. The correlation between the micro and macro-components in olive oil and technological variables are examined using two main chemometric tools, principle component analysis (PCA), and independent components analysis (ICA).

\section{Methodology}

\subsection{Sampling}

A total of seventy-eight olive oil samples of Soury variety were collected during the years 2016 and 2017. The samples were divided into two groups, uncontrolled and controlled.

The uncontrolled group is composed of 58 extra virgin olive oil (EVOO) and virgin olive oil (VOO) samples $(500 \mathrm{ml})$ collected from nine sampling-locations across Lebanon. These locations include Akkar, Hasbaya, Bint Jbail, Tyr, Marjaayoun, Batroun, Koura, Nabatiyeh, and Zgharta and are identified by the EU to have the potential for geographical indications (United Nations [UN], 2010). These samples are referred to as uncontrolled since the olive oil samples were directly collected from farmers (Table 1).

Table 1. Practices applied before, during and after extraction of olive oil

\begin{tabular}{llllll}
\hline & $\begin{array}{l}\text { Harvesting } \\
\text { Date }\end{array}$ & $\begin{array}{l}\text { Olive Fruit } \\
\text { Storage }\end{array}$ & $\begin{array}{l}\text { Fruit storage } \\
\text { duration (days) }\end{array}$ & Extraction method & $\begin{array}{l}\text { Olive } \\
\text { Storage }\end{array}$ \\
\hline Uncontrolled & $\begin{array}{l}\text { Mid-End } \\
\text { October }\end{array}$ & Plastic Bags & $2-3$ days & $\begin{array}{l}\text { Granite Stone/Pressing } \\
\text { mats/ Vertical Centrifuge }\end{array}$ & $\begin{array}{l}\text { Stainless steel } \\
\text { container }\end{array}$ \\
$\begin{array}{l}\text { Carly - Entrolled } \\
\text { October }\end{array}$ & Crates & < one day & $\begin{array}{l}\text { Hammer/ } \\
\text { thermomixer/Centrifuge }\end{array}$ & $\begin{array}{l}\text { Dark Glass } \\
\text { bottles }\end{array}$ \\
\hline
\end{tabular}

The remaining 20 samples were extracted from $5 \mathrm{~kg}$ of healthy Soury olive fruits of a maturity index of 1.76 based on the Uceda et al. (1975) procedure. The fruits were carefully hand-harvested, excluding infected or physically damaged fruits, from randomly selected olive trees in olive orchards located in the above-stated regions. The latter set of samples will be referred to as controlled.

The controlled samples serve to highlight two hypotheses. Firstly, we are interested in proving that the variability emerging from uncontrolled samples does not affect the discrimination among the regions. Secondly, we want to evaluate the impact of technological factors on the chemical and quality parameters compared to those of the uncontrolled extraction.

In this study, regions with similar altitudes across the south and north of Lebanon were grouped into two zones (Figure 1). Zone A (ZA) contains areas with low altitudes ranging from $0 \mathrm{~m}$ to $450 \mathrm{~m}$. Zone B (ZB) contains olive groves situated at high altitudes ranging from $450 \mathrm{~m}$ to $800 \mathrm{~m}$. The climate conditions of the two zones were obtained from the Lebanese Agricultural Research Institute and are presented in Table 2.

Table 2. Sample distribution and climatic conditions of the two zones recorded over two years (2016 and 2017)

\begin{tabular}{llllllll}
\hline & \multicolumn{3}{l}{ Climatic conditions 2016 } & \multicolumn{3}{l}{ Climatic conditions 2017 } \\
\hline Zones & $\begin{array}{l}\text { Altitude } \\
(\mathrm{m})\end{array}$ & $\begin{array}{l}\text { Mean } \\
\text { Precipitation } \\
(\mathrm{mm})\end{array}$ & $\begin{array}{l}\text { Average } \\
\text { Temperature } \\
\left({ }^{\circ} \mathrm{C}\right)\end{array}$ & $\begin{array}{l}\text { Mean } \\
\text { Relative } \\
\text { Humidity } \\
(\%)\end{array}$ & $\begin{array}{l}\text { Mean } \\
\text { Precipitation } \\
(\mathrm{mm})\end{array}$ & $\begin{array}{l}\text { Average } \\
\text { Temperature } \\
\left({ }^{\circ} \mathrm{C}\right)\end{array}$ & $\begin{array}{l}\text { Mean } \\
\text { Relative } \\
\text { Humidity } \\
(\%)\end{array}$ \\
$\mathrm{ZA}^{\dagger}$ & $0-450$ & 578 & 19 & 61 & 501 & 20 & 62 \\
$\mathrm{ZB}^{\ddagger}$ & $450-800$ & 781 & 16 & 48 & 702 & 17 & 54 \\
\hline
\end{tabular}

${ }^{\dagger}$ Number of samples collected from low-altitude regions over the two seasons: Koura (9), Zgharta (9), Tyre (6), Nabatiyeh (7), Batroun (2) and Akkar (4). ${ }^{*}$ Number of samples collected from high-altitude regions over the two 
seasons: Marjaayoun (9), Hasbaya (8), Bint Jbayl (9), Akkar (9) and Batroun (6)

\subsection{Olive Oil Extraction}

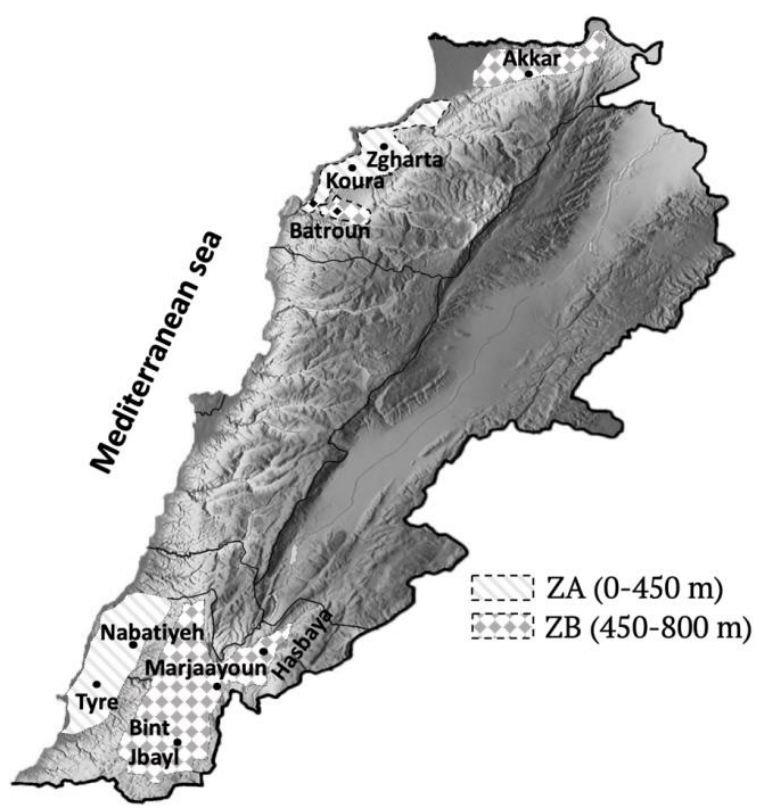

Figure 1. Lebanese map specifying the location of the two zones and the sites where olive fruits and oils were collected

The oil was obtained by cold extraction within $24 \mathrm{~h}$ from harvesting using an Abencor analyzer (Mc2 Ingenieria y Sistemas, Seville, Spain), simulating commercial oil-extraction systems. Olives were crushed using a hammer mill equipped with a $5.5 \mathrm{~mm}$ sieve. The resulting olive paste was distributed into stainless steel containers and kneaded in a mixer at $50 \mathrm{rpm}$ for $30 \mathrm{mins}$ at $28^{\circ} \mathrm{C}$. The obtained paste was then centrifuged at $3500 \mathrm{rpm}$ for 2 mins. All oil samples were decanted and stored in glass bottles at $4^{\circ} \mathrm{C}$ without headspace until analysis.

\subsection{Quality Indicators Determination}

Oil quality indexes including acidity as a percentage of oleic acid (\%), peroxide value (PV) expressed in milliequivalents of active oxygen per kilogram of oil (meq O2/kg), and UV spectrophotometric indices (K232, K270) were determined according to the European Union Commission Regulation EEC No 2568/91 (European Union Commission [EEC], 2013). Spectrophotometric determinations were made using a UV spectrophotometer (Hitachi U-2900, Spectrophotometer, Japan).

\subsection{Fatty Acid Analysis}

The fatty acid methyl esters (FAME) of olive oil were extracted according to the International Olive Council (IOC) method COI/T.20/Doc. No 33 ( IOC, 2017). The FAME was prepared according to Part I and Part II of this method.

A gas chromatograph Shimadzu GC-2025 (Kyoto, Japan) equipped with a capillary column SP-2380 $(30 \mathrm{~m} \times$ $0.32 \mathrm{~mm}$ i.d. x $0.20 \mu \mathrm{m}$ film thickness; Supelco, Bellefonte, PA, USA) and a flame ionization detector was used for the determination of fatty acid profile. Helium was employed as the carrier gas at a flow rate of $1 \mathrm{~mL} / \mathrm{min}$. Injector and detector temperatures were held at $230^{\circ} \mathrm{C}$ and $240^{\circ} \mathrm{C}$, respectively. The injection volume into a split GC port was one $\mu \mathrm{L}$, and a split injection mode (1/100) was used. The following oven temperature program was used: initial temperature $165^{\circ} \mathrm{C}$ held for $10 \mathrm{~min}$; ramped at $1.5^{\circ} \mathrm{C} \mathrm{min}{ }^{-1}$ up to $200^{\circ} \mathrm{C}$. Fatty acids were identified by comparing the retention time of experimental peaks with those obtained by the external standard mixture.

\subsection{Statisitcal Analysis}

\subsubsection{One-way Analysis}

ANOVA was applied to the chemical parameters of olive oil to study the effect of the growing area on the oil characteristics. Also, ANOVA was carried out on the independent components scores to establish statistical 
interpretation. Tukey's test was used to establish the significance of difference at a $5 \%$ level among means.

\subsubsection{Principle Component Analysis}

PCA was developed by Harold Hotelling in the 1930s (Hotelling, 1936). The core idea behind PCA is to represent the initial data matrix by a product of two smaller matrices, the scores matrix $(\mathrm{T})$, and loadings matrices respectively $(\mathrm{P})$ in such a way the product of:

$$
\mathrm{TP}^{\mathrm{t}}+\mathrm{E}=\mathrm{X}
$$

With $\mathrm{P}^{t}$ corresponds to the transposed matrix of $\mathrm{P}$, and $\mathrm{E}$ is the residues matrix.

$\mathrm{T}$ and $\mathrm{P}$ matrices describe the original data in a lower-dimensional space to get a more readable overview of the data. Columns of $\mathrm{T}$ are factorial coordinates of samples (called scores), and lines of $\mathrm{P}^{\mathrm{t}}$ are factorial contributions of variables (called loadings) (Cordella, 2012).

\subsubsection{Independent Component Analysis}

ICA was developed in the 1990s (Comon, 1994). ICA is known primarily as a method of blind source separation (BSS) and can recover $\mathrm{N}$ unknown source signals mixed together, in unknown proportions, in a set of $\mathrm{P}$ observed mixtures. A key point of ICA is that each observation is a mixture of these sources. In most cases, including the case of analytical chemistry data, it is assumed that the part related to the non-linear mixed signals is negligible compared to the linear portion, and we work with a linear mathematical model, which simplifies calculations. This BSS method relies on the assumption that the source signals are statistically independent.

The general model of ICA is:

$$
\mathrm{X}=\mathrm{AS}+\mathrm{E}
$$

where $\mathrm{X}$ is the matrix of the recorded signals, $\mathrm{S}$ is the matrix of "pure" source signals, and A is the unknown mixing matrix corresponding to the "proportions" ("scores") of source signals in each mixture. In recent years, the number of applications of the ICA technique has increased significantly in analytical chemistry, and especially in the treatment of signals acquired during food analysis (Ammari et al., 2012).

Data were analyzed using Matlab version R2015b (The Mathworks Inc., MA, USA).

\section{Results and Discussion}

\subsection{Growing Area Effect on Lebanese Olive Oil}

\subsubsection{Fatty Acid Composition \& Quality Indices}

Table 3 shows the fatty acid composition and quality indices of uncontrolled and controlled samples for ZA and ZB.

The main olive growing areas in Lebanon are mainly distributed along ZA and ZB (Figure 1). Regions belonging to ZA, located on low altitude areas, had higher palmitic, palmitoleic, linoleic, linolenic, and lower oleic acid content when compared to ZB high-altitude regions for both uncontrolled and controlled (Table 3). This is due to the growing area effect, as stated by studies published earlier (Stefanoudaki et al., 1999; Issaoui et al., 2010). For instance, ZA scored a linoleic acid content of $11.37 \%$, a linolenic acid content of $0.64 \%$, and an oleic acid content of $69.94 \%$. As for ZB, an increase in oleic acid (73.24\%) and a decrease in linoleic acid and linolenic acid were observed $(10.46 \%, 0.59 \%)$. These results are per the outcomes described by other studies for oils extracted from olives grown at different altitudes. For instance, Aguilera et al. (2005) showed that oils obtained from high altitudes have a greater content of oleic acid, whereas, at low altitudes, the oils have higher linoleic content, which is the case for ZA and ZB for both controlled and uncontrolled.

In Lebanon, high-altitude areas have a cooler climate compared to low-altitude areas (Table 2). The temperature has an impact on the fatty acid profile of olive oil, as established by several authors (Di Bella et al., 2007; García-Inza et al., 2014). High temperatures increase the content of several fatty acids, such as palmitic, palmitoleic, linoleic and, linolenic, and decrease that of oleic acid. This observation is also noted in ZA and ZB of both groups.

Regarding the quality indices, only the acidity of the samples belonging to ZA showed a significant difference from ZB in the uncontrolled group (Table 3). However, the acidity of ZA and ZB of controlled samples showed no significant difference meaning that this quality parameter is not affected by the growing area. The increase in acidity noticed in ZA may be due to several factors such infestation by pests (Daane \& Johnson, 2010), fungal pathogens (Gomes et al., 2012), advanced ripening (Inglese et al., 2011), and tree fruit load (Trentacoste et al., 2010). Peroxide value, K232, and K270 showed no significant difference between all three zones. 
Table 3. Mean values \pm standard deviation of fatty acids composition and quality indices of Lebanese Olive Oil

\begin{tabular}{|c|c|c|c|c|}
\hline & Uncontrolled & & Controlled & \\
\hline Fatty Acids (\%) and Quality indices & $\mathrm{ZA}^{1}$ & $\mathrm{ZB}^{2}$ & $\mathrm{ZA}$ & ZB \\
\hline Palmitic acid & $11.51 \pm 1.00 \mathrm{~b}$ & $10.46 \pm 1.29 \mathrm{a}$ & $11.63 \pm 0.99 b$ & $9.39 \pm 0.76 a$ \\
\hline Palmitoleic acid & $0.55 \pm 0.09 b$ & $0.46 \pm 0.06 a$ & $0.54 \pm 0.08 b$ & $0.39 \pm 0.06 \mathrm{a}$ \\
\hline Stearic acid & $4.21 \pm 0.39 \mathrm{a}$ & $4.10 \pm 0.31 \mathrm{a}$ & $4.10 \pm 0.40 \mathrm{a}$ & $4.11 \pm 0.38 \mathrm{a}$ \\
\hline Oleic acid & $69.94 \pm 1.44 a$ & $73.14 \pm 1.77 b$ & $71.59 \pm 0.98 a$ & $75.21 \pm 2.02 b$ \\
\hline Linoleic acid & $11.37 \pm 1.24 b$ & $9.83 \pm 1.16 \mathrm{a}$ & $8.97 \pm 1.32 b$ & $8.14 \pm 1.01 \mathrm{a}$ \\
\hline Linolenic acid & $0.64 \pm 0.06 b$ & $0.59 \pm 0.07 \mathrm{a}$ & $0.63 \pm 0.08 b$ & $0.53 \pm 0.05 \mathrm{a}$ \\
\hline $\operatorname{Acidity}^{\dagger}(\mathrm{g} / 100 \mathrm{~g})$ & $1.20 \pm 0.38 b$ & $0.89 \pm 0.43 \mathrm{a}$ & $0.29 \pm 0.04 a$ & $0.32 \pm 0.06 \mathrm{a}$ \\
\hline $\mathrm{PV}^{\ddagger}\left(\right.$ meq $\left.\mathrm{O}_{2} / \mathrm{kg}\right)$ & $11.84 \pm 5.20 \mathrm{a}$ & $11.47 \pm 3.65 \mathrm{a}$ & $2.98 \pm 0.87 a$ & $3.16 \pm 1.27 \mathrm{a}$ \\
\hline K232 & $2.07 \pm 0.34 \mathrm{a}$ & $1.94 \pm 0.23 \mathrm{a}$ & $1.82 \pm 0.24 \mathrm{a}$ & $1.81 \pm 0.19 \mathrm{a}$ \\
\hline K270 & $0.15 \pm 0.15 \mathrm{a}$ & $0.20 \pm 0.14 \mathrm{a}$ & $0.16 \pm 0.03 \mathrm{a}$ & $0.19 \pm 0.05 \mathrm{a}$ \\
\hline
\end{tabular}

All determinations were carried out in duplicate. Different letters in the same row show significant differences (Tukey's test, $\mathrm{p} \leq 0.05$ ). $\uparrow$ Calculated as oleic acid; $₫$ Peroxide value; ${ }^{1}$ olive oil samples collected a low-altitudes; ${ }^{2}$ olive oil samples collected at high-altitudes.

\subsubsection{Exploring the Regional Growing Effects on Fatty Acids by PCA}

A PCA model with 58 uncontrolled olive oil samples and 10 variables, i.e., an initial X data matrix of $58 \times 10$ was conditioned. All data were normalized before running PCA, so all variables have the same standard deviation and thus the same weight. Six principal components (PCs) were extracted, covering $93.7 \%$ of the total variance, where the first two components accounted for $63 \%$ of the variability in the data set. The number of PC components was determined using the scree plot.

The first two PCs were plotted showing a good differentiation between samples located in ZA and ZB associated to different altitudes, average temperatures (Tavg), relative humidity (RH), and precipitation as well as fatty acid profile (Figure 2).

PC1 clearly shows the discrimination of uncontrolled samples belonging to ZA and ZB (Figure 2). Most of the regions belonging to ZB are mostly correlated with oleic acid content and inversely correlated with RH. It has been reported by Ranalli et al. (1997) that the percentage of oleic acid is negatively correlated with the RH of the atmosphere. This coincides with the fact that most of the regions in ZA are characterized by RH and thus less influenced by oleic content. Another reason for such low content of oleic acid in ZA is the altitude. Several authors demonstrated that olives grown at lower altitudes with high average temperatures showed lower oleic acid and higher polyunsaturated and saturated fatty acids in contrary to those grown at higher altitudes with cooler climate (Issaoui et al., 2010; Stefanoudaki et al., 1999). Also, García-Inza et al. (2014) studied the effect of high temperature by placing olive fruiting branches in transparent plastic chambers with individualized temperature control. The results showed that high temperatures decrease the oleic acid content and increase the palmitic, palmitoleic, linoleic, and linoleic acid contents. So, the scores and loadings of ZA and ZB on PC1 are in agreement with the information given above. ZA has positive scores and is correlated with the following variables: linoleic, linolenic, palmitic, palmitoleic, temperature, and $\mathrm{RH}$, whereas $\mathrm{ZB}$ scores were negative and correlate d with oleic, and altitude parameters respectively (Figure 2). It is noteworthy to mention that the variable "precipitation" did not affect the discrimination between ZA and ZB since the distance between the latter and the origin is closer than all other variables.

As ZA and ZB represent the main olive growing areas in this study, the above PCA model needs to be validated since it was conducted on uncontrolled samples. So, a set of 20 samples (Controlled) with optimum conditions were projected on the above PCA model. The new observations seem to fit the above discrimination based on PC1 (Figure 3). ZB is still dominated by oleic acid content, whereas ZA is characterized by linoleic, linolenic, palmitoleic, and palmitic acid. The climatic conditions responsible for such discrimination are altitude, temperature, and RH. This is the same result obtained with the uncontrolled samples.

Apparently, the growing area had a significant impact on fatty acids, even in the presence of other forms of variability, such as processing techniques and different oil qualities. 


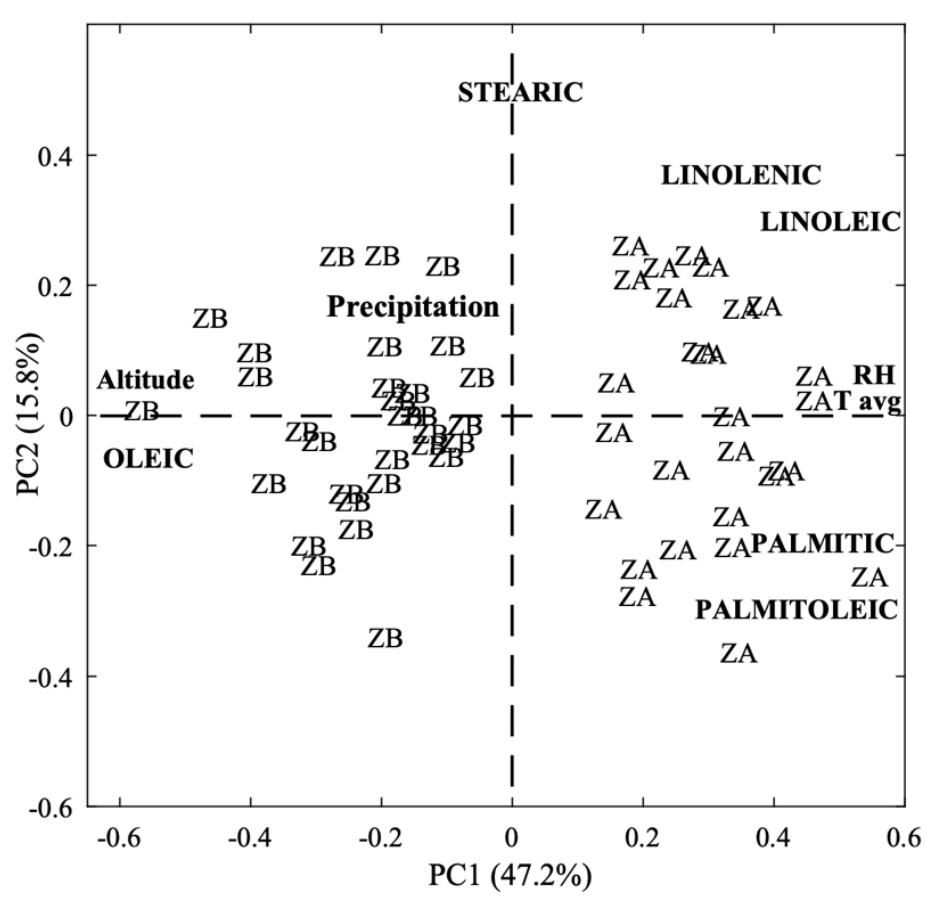

Figure 2. Principal component analysis of uncontrolled olive oil samples showing the distribution of the two zones based on fatty acid profiles. ZA: Zone A (olive oil samples collected at low altitudes); ZB; Zone B (olive oil samples collected at high altitudes)

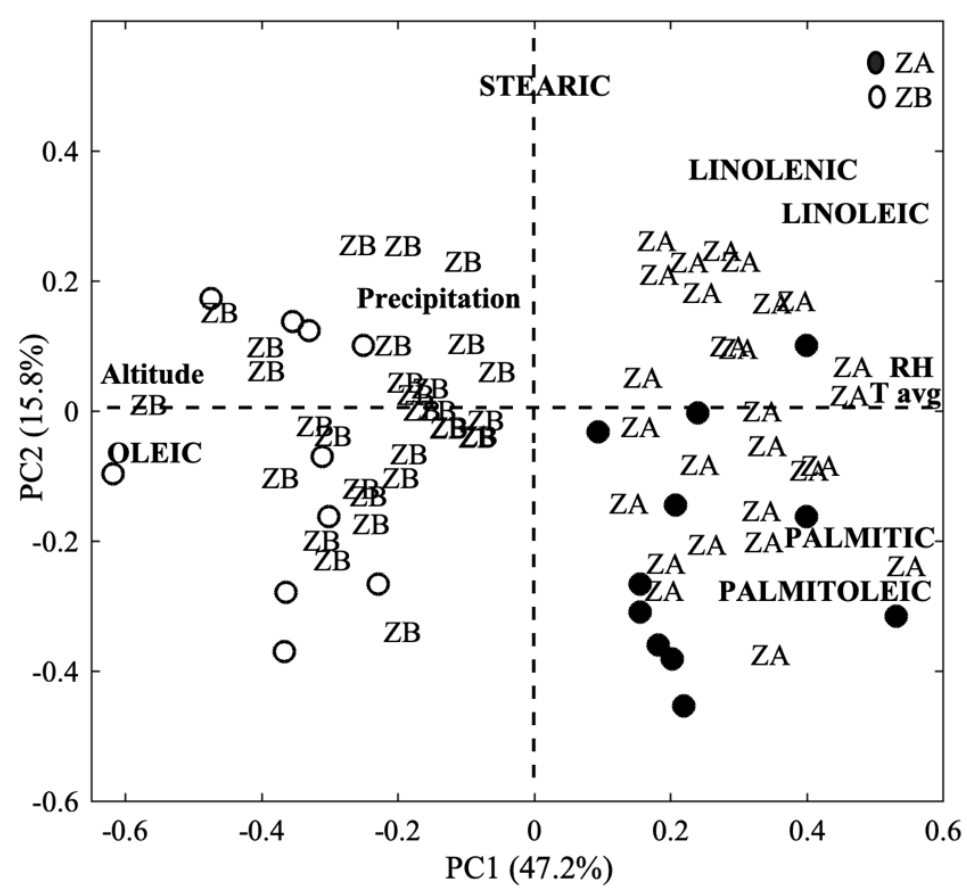

Figure 3. Bi-plot presentation showing the projection of the observations of controlled olive oil samples on the uncontrolled PCA. ZA: Zone A (olive oil samples collected at low altitudes), ZB: Zone B (olive oil samples collected at high altitudes)

\subsection{Factors Causing the Disqualification of Olive Oil Virginity by ICA}

Several crucial parameters for obtaining a good quality olive oil were studied to detect the leading factor in the 
disqualification of Lebanese olive oil from being considered an extra virgin. In 2016, 25 thousand tons of olive oil were produced, and only $16 \%$ (3929 tons) of total olive oil production consisted of EVOO (IDAL, 2017). To assess the above situation, a comparison was established between the uncontrolled group and the controlled group. It is noteworthy to mention that in this comparison, 20 samples that share the same altitude and climatic conditions as those of the controlled group were selected from the total number of uncontrolled samples. In this study, ICA combined with one-way ANOVA was chosen over PCA since the analysis of the loadings carried out by the latter is often not direct, and may highlight mixtures of different phenomena explained by the data (Rutledge \& Jouan-Rimbaud Bouveresse, 2013). Data were standardized and an ICA model with 4 independent components (IC) was calculated from the dataset. Then the proportions of each IC component were subjected to one-way ANOVA to establish the statistical inference.

The ICs corresponding to the separation of both groups based on the significance level $(p<0.05)$ are IC1 $(3.34 \mathrm{E}-10<0.05)$, IC2 $(2.30 \mathrm{E}-06<0.05)$, IC3 $(4.99 \mathrm{E}-06<0.05)$, IC4 $(8.40 \mathrm{E}-08<0.05)$, which are in turn attributed to peroxide value, oleic acid, linoleic acid, and acidity, respectively. Figure 4 shows the proportions of the significant IC components (equivalent scores), and their corresponding pure signals (unmixed loadings).
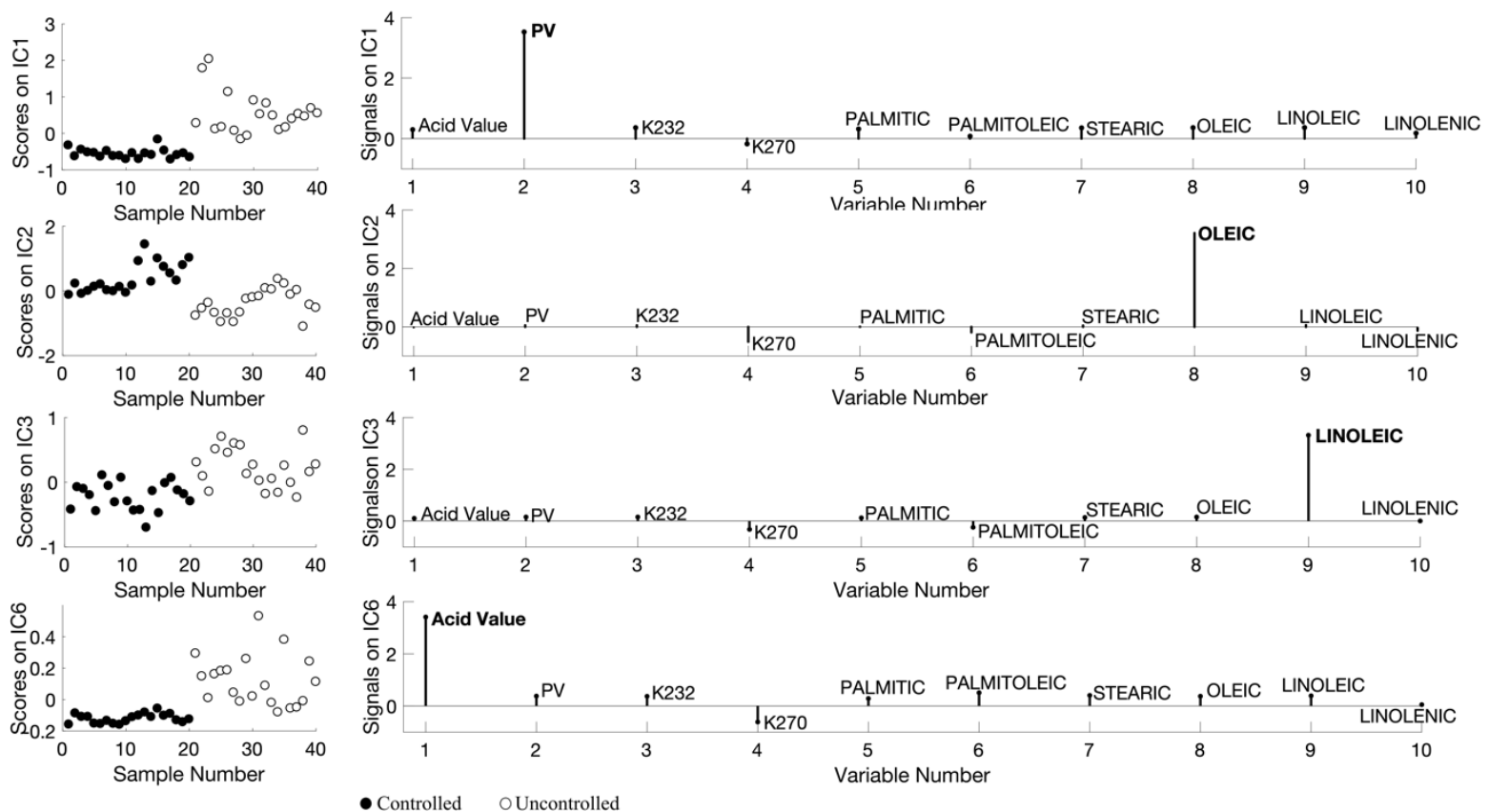

Figure 4. ICA scores and loadings of Lebanese olive oil chemical parameters; PV: Peroxide value.

ANOVA was also conducted on the above groups as an extra measure of certainty for the ICA results. The key variables obtained by ICA for differentiating both groups are in coordination with those obtained with ANOVA, as shown in Table 4. The uncontrolled group, classified as VOO (according to IOC standards), exhibited significantly higher acidity and peroxide values when compared with oils extracted under optimum conditions. In addition, lower content of oleic and higher content of linoleic acid was observed in the uncontrolled group. As for the controlled group, they are classified as EVOO since all quality parameters are within the norms set by IOC for EVOO (Table 4). Several factors and practices interfere in affecting olive oil quality and composition, varying from agricultural to technological ones (Gharbi et al., 2015). Based on Table 1, the two critical factors/practices responsible for the quality deterioration of olive oil are fruit storage condition and duration, and extraction method.

Although in the comparison mentioned earlier, modern processing technique was compared against the traditional ones (Table 1), the latter technique should manage to produce EVOO. Several authors reported that pressing systems can produce EVOO if the machinery and the production site are clean (Di Giovacchino et al., 1994; Di Giovacchino et al., 1996). Besides, IOC, in its guide on quality management for the olive oil industry, recommends the application of good hygienic and manufacturing practices to ensure the best olive oil quality outcome (IOC, 2006). Most of the traditional mills in Lebanon do not apply any means for cleanliness or quality control. Olive paste in layers stacked on pressing mats, which are challenging to be kept clean and exempt from 
oxidized oil and fermentation defects possibly transferable to the oils. Besides, most of the farmers transport olive fruits in plastic sacks that stay for several days in the mill before being pressed. These harmful practices favor the enzymatic lipolysis of the fruit triacylglycerol, which strongly affects the quality indicators of olive oil (Kiritsakis et al., 1998).

Table 4. Fatty acid composition and quality indices of olive oil obtained either by controlled or uncontrolled extraction

\begin{tabular}{lll}
\hline Fatty Acids (\%) and Quality indices & Uncontrolled & Controlled \\
\hline Palmitic acid & $10.95 \pm 1.27 \mathrm{a}$ & $10.51 \pm 1.40 \mathrm{a}$ \\
Palmitoleic acid & $0.50 \pm 0.09 \mathrm{a}$ & $0.46 \pm 0.10 \mathrm{a}$ \\
Stearic acid & $4.11 \pm 0.35 \mathrm{a}$ & $4.10 \pm 0.30 \mathrm{a}$ \\
Oleic acid & $71.65 \pm 2.28 \mathrm{a}$ & $73.40 \pm 2.36 \mathrm{~b}$ \\
Linoleic acid & $10.54 \pm 1.43 \mathrm{~b}$ & $8.56 \pm 1.19 \mathrm{a}$ \\
Linolenic acid & $0.61 \pm 0.07 \mathrm{a}$ & $0.58 \pm 0.08 \mathrm{a}$ \\
Acidity $\dagger(\mathrm{g} / 100 \mathrm{~g})$ & $1.04 \pm 0.43 \mathrm{~b}$ & $0.30 \pm 0.05 \mathrm{a}$ \\
$\mathrm{PV} \ddagger(\mathrm{meq} \mathrm{O2} / \mathrm{kg})$ & $11.64 \pm 4.40 \mathrm{~b}$ & $3.07 \pm 1.07 \mathrm{a}$ \\
K232 & $1.94 \pm 0.27 \mathrm{a}$ & $1.81 \pm 0.20 \mathrm{a}$ \\
K270 & $0.18 \pm 0.07 \mathrm{a}$ & $0.18 \pm 0.04 \mathrm{a}$ \\
\hline Percentage of samples classified as EVOO & $100 \%$ & $33.30 \%$ \\
\hline
\end{tabular}

All determinations were carried out in duplicate and mean value \pm standard deviation. Different letters in the same row show significant differences $(\mathrm{p} \leq 0.05)$. $\uparrow$ Calculated as oleic acid; $₫$ Peroxide value.

\section{Conclusion}

PCA and one-way ANOVA were able to reveal a correlation between the fatty acid profile and the pedoclimatic conditions of the main olive growing regions in Lebanon. Low altitudes regions were characterized by palmitic, palmitoleic, linoleic, and linolenic acid, whereas high-altitude regions were represented by their high oleic acid content.

On the other hand, ICA facilitated the chemical interpretation by providing chemically pure components that crossed the parameters sets by IOC for EVOO. Fruit storage condition/duration and the non-hygienic conditions of the extraction method were identified as the major quality affecting parameters. The solution for such non-compliances would rely on good management/hygienic practices which include, ensuring suitable pre-processing and post-processing storage conditions and providing basic hygiene.

\section{Acknowledgments}

The authors would like to acknowledge the National Council for Scientific Research of Lebanon [5/2106] and the Lebanese University [504/12/CU] for their financial support and for granting a doctoral fellowship to "Omar Dib". We also thank all olive farmers; whose assistance was essential to this study execution. All authors contributed equally in experiment conception, conduction, data analysis and manuscript writing and final correction.

\section{References}

Aguilera, M. P., Beltrán, G., Ortega, D., Fernández, A., Jiménez, A., \& Uceda, M. (2005). Characterisation of virgin olive oil of Italian olive cultivars: 'Frantoio' and 'Leccino', grown in Andalusia. Food Chemistry, 89(3), 387-391. https://doi.org/10.1016/j.foodchem.2004.02.046

Amanpour, A., Kelebek, H., \& Selli, S. (2019). Characterization of aroma, aroma-active compounds and fatty acids profiles of cv. Nizip Yaglik oils as affected by three maturity periods of olives. Journal of the Science of Food and Agriculture, 99(2), 726-740. https://doi.org/10.1002/jsfa.9241

Ammari, F., Jouan-Rimbaud-Bouveresse, D., Boughanmi, N., \& Rutledge, D. N. (2012). Study of the heat stability of sunflower oil enriched in natural antioxidants by different analytical techniques and front-face fluorescence spectroscopy combined with Independent Components Analysis. Talanta, 99, 323-329. https://doi.org/10.1016/j.talanta.2012.05.059

Aparicio, R., Ferreiro, L., \& Alonso, V. (1994). Effect of climate on the chemical composition of virgin olive oil. Analytica Chimica Acta, 292(3), 235-241. https://doi.org/10.1016/0003-2670(94)00065-4

Arslan, D., Karabekir, Y., \& Schreiner, M. (2013). Variations of phenolic compounds, fatty acids and some 
qualitative characteristics of Sarıulak olive oil as induced by growing area. Food Research International, 54(2), 1897-1906. https://doi.org/10.1016/j.foodres.2013.06.016

Comon, P. (1994). Independent component analysis, A new concept?. Signal Processing, 36(3), 287-314. https://doi.org/10.1016/0165-1684(94)90029-9

Cordella, C. B. Y. (2012). Prinicple Component Analysis: The Basic Building Block of Chemometrics. L'Actualité Chimique, 13-18. https://doi.org/10.5772/51429

Daane, K. M., \& Johnson, M. W. (2010). Olive fruit fly: managing an ancient pest in modern times. Annu Rev Entomol, 55, 151-169. https://doi.org/10.1146/annurev.ento.54.110807.090553

Di Bella, G., Maisano, R., La Pera, L., Lo Turco, V., Salvo, F., \& Dugo, G. (2007). Statistical Characterization of Sicilian Olive Oils from the Peloritana and Maghrebian Zones According to the Fatty Acid Profile. Journal of Agricultural and Food Chemistry, 55(16), 6568-6574. https://doi.org/10.1021/jf070523r

Di Giovacchino, L., Angerosa, F., \& Di Giacinto, L. (1996). Effect of mixing leaves with olives on organoleptic quality of oil obtained by centrifugation. Journal of the American Oil Chemists' Society, 73(3), 371-374. https://doi.org/10.1007/bf02523433

Di Giovacchino, L., Solinas, M., \& Miccoli, M. (1994). Effect of extraction systems on the quality of virgin olive oil. Journal of the American Oil Chemists'Society, 71(11), 1189-1194. https://doi.org/10.1007/bf02540535

EEC. (2013). Implementing Regulation No 1348/2013 amending Regulation (EEC) No 2568/91 on the characteristics of olive oil and olive-residue oil and on the relevant methods of analysis. Official Journal of the European Union, 31-67.

García-Inza, G. P., Castro, D. N., Hall, A. J., \& Rousseaux, M. C. (2014). Responses to temperature of fruit dry weight, oil concentration, and oil fatty acid composition in olive (Olea europaea L. var. 'Arauco'). European Journal of Agronomy, 54, 107-115. https://doi.org/10.1016/j.eja.2013.12.005

Gharbi, I., Issaoui, M., Mehri, S., Cheraief, I., Sifi, S., \& Hammami, M. (2015). Agronomic and Technological Factors Affecting Tunisian Olive Oil Quality. Agricultural Sciences, 06(15). https://doi.org/10.4236/as.2015.65051

Giovacchino, L. D. (2000). Technological Aspects: Handbook of Olive Oil: Analysis and Properties. Gaithersburg, MD (USA): Aspen Publisher, 17-59. https://doi.org/10.1007/978-1-4757-5371-4_2

Gomes, S. n., Prieto, P., Carvalho, T., Guedes-Pinto, H., \& Martins-Lopes, P. (2012). Olive - Colletotrichum acutatum: An Example of Fruit-Fungal Interaction. Rijeka, Croatia: IntechOpen (CY). https://doi.org/10.5772/27965

Hotelling, H. (1936). Relations Between Two Sets of Variates. Biometrika, 28, 321-377. https://doi.org/10.1093/biomet/28.3-4.321

IDAL. (2017). Olive Oil Industry in Lebanon. Investment Development Authority in Lebanon. Retrieved from https://investinlebanon.gov.lb/Content/uploads/CorporatePageRubric/180123031939637 Olive\%20Oil\%20 factsheet\%202017.pdf

Inglese, P., Famiani, F., Galvano, F., Servili, M., Esposto, S., \& Urbani, S. (2011). Factors Affecting Extra-Virgin Olive Oil Composition: Horticultural Reviews. Hoboken, New Jersey: Wiley-Blackwell, 83-147. https://doi.org/10.1002/9780470872376.ch3

IOC. (2006). Quality management guide for the olive oil industry: olive oil mills. International Olive Council. T.33/Doc. No. 2-4.

IOC. (2017). Method of Analysis, Determination of fatty acid methyl esters by gas chromatography. International Olive Council. COI/T.20/Doc. No. 33

IOC. (2018). World olive oil figures. International Olive Council. Retrieved from http://www.internationaloliveoil.org/estaticos/view/131-world-olive-oil-figures

Issaoui, M., Flamini, G., Brahmi, F., Dabbou, S., Hassine, K. B., Taamali, A., Chehab, H., Ellouz, M., Zarrouk, M. ... Hammami, M. (2010). Effect of the growing area conditions on differentiation between Chemlali and Chétoui olive oils. Food Chemistry, 119(1), 220-225. https://doi.org/10.1016/j.foodchem.2009.06.012

Kiritsakis, A., Nanos, G. D., Polymenopulos, Z., Thomai, T., \& Sfakiotakis, E. M. (1998). Effect of fruit storage conditions on olive oil quality. Journal of the American Oil Chemists' Society, 75(6), 721-724. 
https://doi.org/10.1007/s11746-998-0212-7

Merchak, N., El Bacha, E., Bou Khouzam, R., Rizk, T., Akoka, S., \& Bejjani, J. (2017). Geoclimatic, morphological, and temporal effects on Lebanese olive oils composition and classification: A $1 \mathrm{H}$ NMR metabolomic study. Food Chemistry, 217(3), 79-388. https://doi.org/10.1016/j.foodchem.2016.08.110

MOA. (2012). Overview of the Lebanese Olive Sector Report, Lebanese Republic Ministry of Agriculture.

Ranalli, G., de Mattia, G., Ferrante, M. L., \& Giansante, L. (1997). Incidence of Olive Cultivation Area on the Analytical Characteristics of the Oil. Riv. Ital. Sostanze Grasse, 74, 501-508. https://doi.org/10.1007/s11746-997-0032-1

Riachy, M., Bou, M. C., Youssef, A., Andary, R., \& Skaff, W. (2018). Chemical and Sensorial Characteristics of Olive Oil Produced from the Lebanese Olive Variety 'Baladi'. Sustainability, 10, 1-21. https://doi.org/10.3390/su10124630

Rutledge, D. N., \& Jouan-Rimbaud, B. D. (2013). Independent Components Analysis with the JADE algorithm. TrAC Trends in Analytical Chemistry, 50, 22-32. https://doi.org/10.1016/j.trac.2013.03.013

Stefanoudaki, E., Kotsifaki, F., \& Koutsaftakis, A. (1999). Classification of virgin olive oils of the two major cretan cultivars based on their fatty acid composition. Journal of the American Oil Chemists' Society, 76(5), 623-626. https://doi.org/10.1007/s11746-999-0013-7

Trentacoste, E. R., Puertas, C. M., \& Sadras, V. O. (2010). Effect of fruit load on oil yield components and dynamics of fruit growth and oil accumulation in olive (Olea europaea L.). European Journal of Agronomy, 32(4), 249-254. https://doi.org/10.1016/j.eja.2010.01.002

Uceda, M., \& Frías, L. (1975). Evolución del contenido graso del fruto y de la composición y calidad del aceite. IOOC (ed) Proc. of II Seminario Oleícola International. Córdoba, Spain.

\section{Copyrights}

Copyright for this article is retained by the author(s), with first publication rights granted to the journal.

This is an open-access article distributed under the terms and conditions of the Creative Commons Attribution license (http://creativecommons.org/licenses/by/4.0/). 